\title{
Comparison of Various Routing Protocol in Wireless Sensor Network
}

\author{
Parul Kansal \\ M. Tech. [E C E] \\ ECE Deptt. \\ NIT Kurukshetra, INDIA
}

\author{
Deepali Kansal \\ B.Tech [c s]
}

\author{
Arun Balodi \\ M. Tech. [D S P] \\ EEED, GBPEC, Pauri \\ Uttarakhand, INDIA
}

\begin{abstract}
Wireless Sensor Networks have emerged as an important new area in wireless technology. Wireless Sensor Networks (WSN) consists of numerous tiny sensors deployed at high density in regions requiring surveillance and monitoring. There are many existing protocol, techniques and concepts from traditional wireless network, such as cellular network, mobile ad-hoc network, wireless local area network and Bluetooth, are applicable and still used in wireless sensor network, but there are also many fundamental differences which lead to the need of new protocols and techniques. Analysis of various Routing protocols via simulation suitable for WSN. The design of routing protocols for WSN's must consider the power and resource limitation of the network nodes, the time varying quality of wireless channels and possibility of packet loss and delay. To address these design requirements several design strategies for WSN's have been proposed. AODV, DSDV, LEACH, some of the common protocols. Each has its fair share of advantages and limitation.
\end{abstract}

\section{Keywords}

WSN, Routing, AODV, DSDV, LEACH,

\section{INTRODUCTION}

Sensor networks can contain hundreds or thousands of sensing nodes. It is desirable to make these nodes as cheap and energyefficient as possible and rely on their large numbers to obtain high quality results [2]. Network protocols must be designed to achieve fault tolerance in the presence of individual node failure while minimizing energy consumption. In addition, since the limited wireless channel bandwidth must be shared among all the sensors in the network, routing protocols for these networks should be able to perform local collaboration to reduce bandwidth requirements. Communication between the sensor nodes and the base station is expensive, and there are no "high energy" nodes through which communication can proceed [4].

The characteristics of the sensor networks as:

1. The number of sensor nodes in a sensor network can be several orders of magnitude higher than the nodes in an ad hoc network.

2. Sensor nodes are densely deployed.

3. Sensor nodes are prone to failures.

4. The topology of a sensor network changes very frequently.
5. Sensor nodes mainly use broadcast communication paradigm

6. Sensor nodes are limited in power, computational capacities, and memory.

7. Sensor nodes may not have global identification (ID) because of the large amount of overhead and large number of sensors.

The application of sensor network is very abroad, such as:

- Military applications

- Environmental applications

- Health applications

- Home applications

- Industrial applications

\section{ROUTING OBJECTIVES}

Some sensor network applications only require the successful delivery of messages between a source and a destination. However, there are applications that need even more assurance. These are the real-time requirements of the message delivery, and in parallel, the maximization of network lifetime [3].

a) Non-real time delivery: The assurance of message delivery is indispensable for all routing protocols. It means that the protocol should always find the route between the communicating nodes, if it really exists. This correctness property can be proven in a formal way, while the averagecase performance can be evaluated by measuring the message delivery ratio.

b) Real-time delivery: Some applications require that a message must be delivered within a specified time, otherwise the message becomes useless or its information content is decreasing after the time bound. Therefore, the main objective of these protocols is to completely control the network delay. The average-case performance of these protocols can be evaluated by measuring the message delivery ratio with time constraints.

c) Network lifetime: This protocol objective is crucial for those networks, where the application must run on sensor nodes as long as possible. The protocols aiming this concern try to balance the energy consumption equally among nodes considering their residual energy levels. However, the metric used to determine the network lifetime is also application dependent. Most protocols assume that 
every node is equally important and they use the time until the first node dies as a metric, or the average energy consumption of the nodes as another metric. If nodes are not equally important, then the time until the last or highpriority nodes die can be a reasonable metric.

\subsection{AODV [Ad-hoc on-demand distance vector]}

AODV is a method of routing messages between mobile computers. It allows these mobile computers, or nodes, to pass messages through their neighbors to nodes with which they cannot directly communicate. AODV does this by discovering the routes along which messages can be passed. AODV makes sure these routes do not contain loops and tries to find the shortest route possible [1]. AODV is also able to handle changes in routes and can create new routes if there is an error. The diagram to the left shows a set up of four nodes on a wireless network. The circles illustrate the range of communication for each node. Because of the limited range, each node can only communicate with the nodes next to it [6].

AODV is one of the most efficient routing protocols in terms of establishing the shortest path and lowest power consumption. It is mainly used for ad-hoc networks but also in wireless sensor networks. It uses the concepts of path discovery and maintenance. However, AODV builds routes between nodes ondemand i.e. only as needed. So, AODVs' primary objectives are:

1. To broadcast discovery packets only when necessary,

2. To distinguish between local connectivity management (neighborhood detection) and general topology maintenance,

3. To disseminate information about changes in local connectivity to those neighboring mobiles nodes that are likely to need the information.

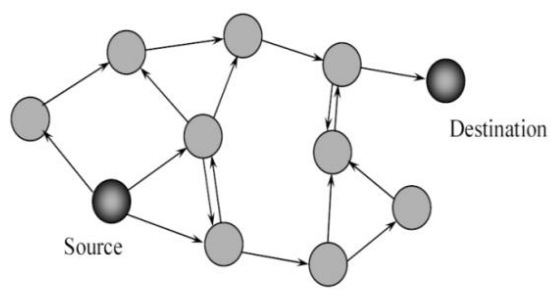

Figure 1: Route Request (RREQ) broadcast flood

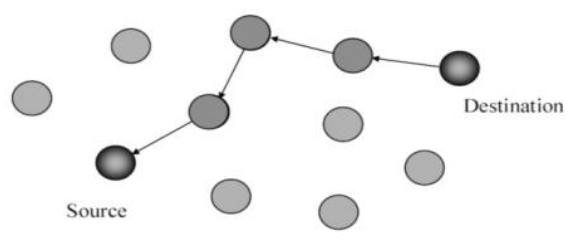

Figure 2: Route Reply (RREP) propagation

\section{AODV Characteristics}

1. Will find routes only as needed.

2. Use of Sequence numbers to track accuracy of information.

3. Only keeps track of next hop for a route instead of the entire route.

4. Use of periodic HELLO messages to track Neighbors.

\section{Pros}

- The AODV routing protocol does not need any central administrative system to control the routing process.

- Reactive protocols like AODV tend to reduce the control traffic messages overhead at the cost of increased latency in finding new routes.

\section{Cons}

- It is possible that a valid route is expired.

- The performance of the AODV protocol without any misbehaving nodes is poor in larger networks.

\section{DSDV [Destination-sequenced distance vector]}

Destination-Sequenced Distance Vector routing protocol (DSDV) is a typical routing protocol is based on the Distributed Bellman-Ford algorithm [3]. In DSDV, each route is tagged with a sequence number which is originated by the destination, indicating how old the route is. Each node manages its own sequence number by assigning it two greater than the old one (call an even sequence number) every time. When a route update with a higher sequence number is received, the old route is replaced. In case of different routes with the same sequence number, the route with better metric is used. Updates are transmitted periodically or immediately when any significant topology change is detected. There are two ways of performing routing update: "full dump", in which a node transmits the complete routing table, and "incremental update", in which a node sends only those entries that have changed since last update. To avoid fluctuations in route updates, DSDV employs a "settling time" data, which is used to predict the time when route becomes stable. In DSDV, broken link may be detected by the layer- 2 protocol or it may instead be inferred if no broadcasts have been received for a while from a former neighboring node.

\section{DSDV Characteristics}

- Proactive - based on Bellman - Ford.

- Packets transmitted according to the routing table.

- Each node maintains routing table with entry for each node in the network.

- Each node maintains its own sequence number.

- Updates at each change in neighborhood information.

- Used for freedom from loops.

- To distinguish stale routes from new ones.

\section{Pros}

- Proactive Routes maintained through periodic and event triggered routing table exchanges.

- All available information is transmitted. 


\section{Cons}

- Frequency of transmitting full updates is reduced if the volume of data begins to consume significant bandwidth.

\subsection{LEACH [Low-energy adaptive clustering hierarchy]}

LEACH is hierarchical routing approach for sensors networks. The idea proposed in LEACH has been an inspiration for many hierarchical routing protocols, although some protocols have been independently developed. We explore hierarchical routing protocols in following section.

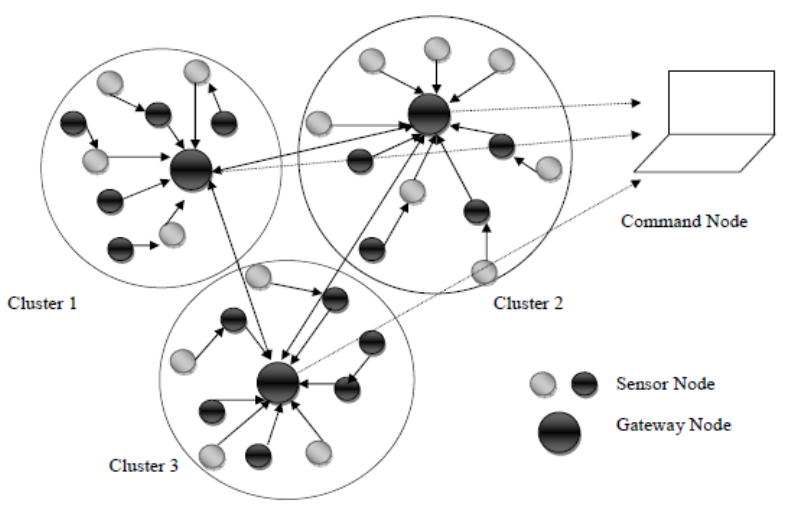

Figure 3: Hierarchical or cluster-based routing

The main aim of hierarchical routing is to efficiently maintain the energy consumption of sensor nodes by involving them in multi-hop communication within a particular cluster and by performing data aggregation and fusion in order to decrease the number of transmitted messages to the sink. Cluster formation is typically based on the energy reserve of sensors and sensor's proximity to the cluster head.

Hierarchical or cluster-based routing, originally proposed in wire line networks, are well-known techniques with special advantages related to scalability and efficient communication. As such, the concept of hierarchical routing is also utilized to perform energy-efficient routing in WSNs. In a hierarchical architecture, higher energy nodes can be used to process and send the information while low energy nodes can be used to perform the sensing in the proximity of the target. This means that creation of clusters and assigning special tasks to cluster heads can greatly contribute to overall system scalability, lifetime, and energy efficiency. Hierarchical routing is an efficient way to lower energy consumption within a cluster and by performing data aggregation and fusion in order to decrease the number of transmitted messages to the BS. Hierarchical routing is mainly two-layer routing where one layer is used to select cluster heads and the other layer is used for routing. LEACH randomly selects a few sensor nodes as cluster heads $(\mathrm{CHs})$ and rotates this role to evenly distribute the energy load among the sensors in the network [9]. In LEACH, the cluster head $(\mathrm{CH})$ nodes compress data arriving from nodes that belong to the respective cluster, and send an aggregated packet to the base station in order to reduce the amount of information that must be transmitted to the base station. LEACH uses a
TDMA/CDMA MAC to reduce inter-cluster and intra-cluster collisions. However, data collection is centralized and is performed periodically. Therefore, this protocol is most appropriate when there is a need for constant monitoring by the sensor network. A user may not need all the data immediately. Hence, periodic data transmissions are unnecessary which may drain the limited energy of the sensor nodes. After a given interval of time, a randomized rotation of the role of the $\mathrm{CH}$ is conducted so that uniform energy dissipation in the sensor network is obtained. The operation of LEACH is separated into two phases, the setup phase and the steady state phase. In the setup phase, the clusters are organized and $\mathrm{CHs}$ are selected. In the steady state phase, the actual data transfer to the base station takes place. The duration of the steady state phase is longer than the duration of the setup phase in order to minimize overhead. During the setup phase, a predetermined fraction of nodes, $\mathrm{p}$, elect themselves as $\mathrm{CHs}$ as follows. A sensor node chooses a random number, $r$, between 0 and 1 . If this random number is less than a threshold value, $\mathrm{T}(\mathrm{n})$, the node becomes a clusterhead for the current round. The threshold value is calculated based on an equation that incorporates the desired percentage to become a cluster-head, the current round, and the set of nodes that have not been selected as a cluster-head in the last $(1 / \mathrm{P})$ rounds, denoted by $\mathrm{G}$. It is given by:

$$
T(n)=\frac{p}{1-p\left(\operatorname{rmod}\left(\frac{2}{D}\right)\right)} \text { if } n \in G
$$

Where $\mathrm{G}$ is the set of nodes that are involved in the $\mathrm{CH}$ election. Each elected $\mathrm{CH}$ broadcast an advertisement message to the rest of the nodes in the network that they are the new cluster-heads. All the non-cluster head nodes, after receiving this advertisement, decide on the cluster to which they want to belong to. This decision is based on the signal strength of the advertisement. The non cluster-head nodes inform the appropriate cluster-heads that they will be a member of the cluster. After receiving all the messages from the nodes that would like to be included in the cluster and based on the number of nodes in the cluster, the cluster-head node creates a TDMA schedule and assigns each node a time slot when it can transmit. This schedule is broadcast to all the nodes in the cluster.

During the steady state phase, the sensor nodes can begin sensing and transmitting data to the cluster-heads. The clusterhead node, after receiving all the data, aggregates it before sending it to the base-station. After a certain time, which is determined a priori, the network goes back into the setup phase again and enters another round of selecting new $\mathrm{CH}$. Each cluster communicates using different CDMA codes to reduce interference from nodes belonging to other clusters.

\section{Pros}

- Distributed and no global knowledge of network required. Cons

- Extra overhead to do dynamic clustering.

\section{RESULT AND DISCUSSIONS}

\subsection{Performance metrics used}

In this section performance metrics are used to evaluate performance of routing protocols and data dissemination 
protocols scheme when no in-networking processing is performed and no caching is used.

- Packet Delivery Ratio

- Routing Over Head (ROH)

- Throughput (Kbps)

- Average End to End Delay (ms)

\subsection{Parameter Used}

Table 1: Simulation Parameter for Routing Protocol

\begin{tabular}{|l|c|}
\hline Parameter & Value \\
\hline Simulation Time & 100 Seconds \\
\hline Terrain Area & $500 \mathrm{~m} \times 400 \mathrm{~m}$ \\
\hline Time Intervals & 20 Seconds \\
\hline Traffic Type & Udp \\
\hline No. of Nodes & $25,50,75,100$ \\
\hline
\end{tabular}

\subsection{Simulation of Routing Protocol}

In this section we evaluate the performance of AODV, DSDV and LEACH protocols on the following parameters for WSNs:

a) Packet Delivery Ratio (PDR)

The ratio between the number of packets that are received and the number of packets sent.

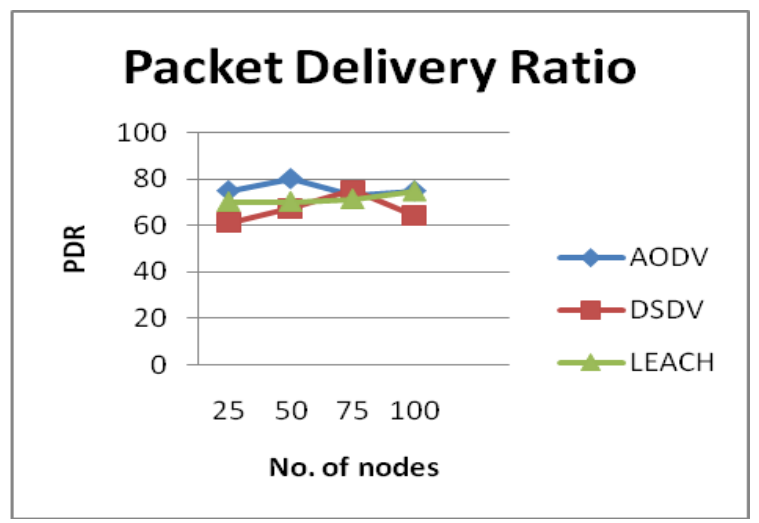

Figure 4: Three protocols of WSNs with respect to PDR

\section{Result:}

1. Based on the figure 4 , it is not possible to say clearly that the PDR in AODV are decreases or increases with respect to number of nodes, because at network size 25 to 50 there is increase but at network size 50 to 75 there is decrease and for network size 75 to 100 again increase, but PDR is higher than DSDV and LEACH, but LEACH shows stability as compared to others.
2. In DSDV, the PDR increases smoothly as number of nodes increases except for network size 100.

3. In LEACH, the PDR increases as number of nodes become larger.

4. The performance of AODV is better with more number of nodes than in comparison with other two protocols.

\section{Packet Delivery Ratio}

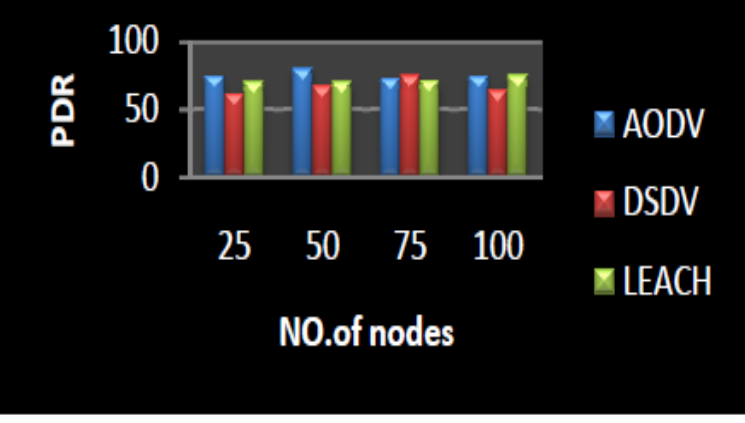

Figure 5: Comparison of the three protocols of WSNs with respect to $P D R$.

b) Routing Over Head (ROH)

The routing overhead measures by the total number of control packets sent divided by the number of data packets delivered successfully.

\section{Routing Over Head}

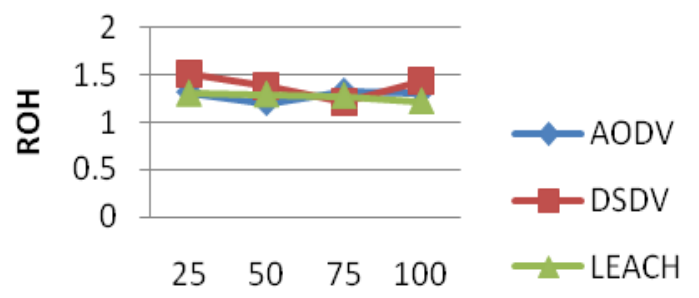

No. of nodes

Figure 6: Three protocols of WSNs with respect to ROH

\section{Result:}

1. From figure 6, in AODV, the result of ROH is increases at some points and decreases at other points like PDR.

2. In DSDV, the ROH decreases as number of nodes increases except network size 100 as like PDR.

3. In $\mathrm{LEACH}$, the $\mathrm{ROH}$ decreases smoothly when the number of nodes increases.

4. The performance of AODV is better with more number of nodes than in comparison with other two protocols. 


\section{Routing Over Head}

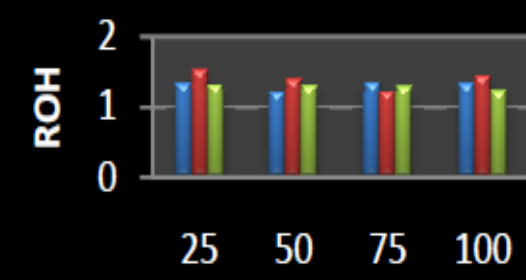

no. of nodes
— AODV

ㅁDSDV

ㄴ.EACH

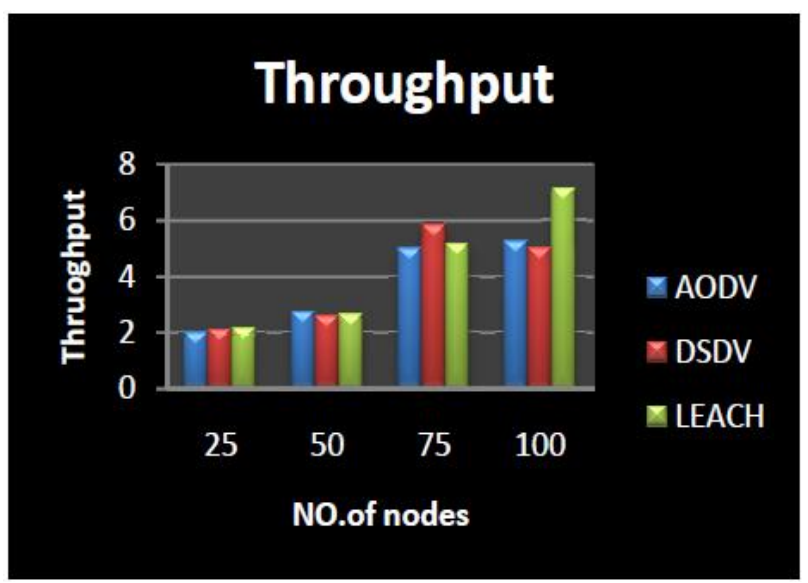

Figure 9: Comparison of the three protocols of WSNs with respect to Throughput

\section{d) Average End-to-End Delay}

This delay includes processing and queuing delay in each intermediate node i.e. the time elapsed until a demanded route is available. Unsuccessful route establishment are ignored.

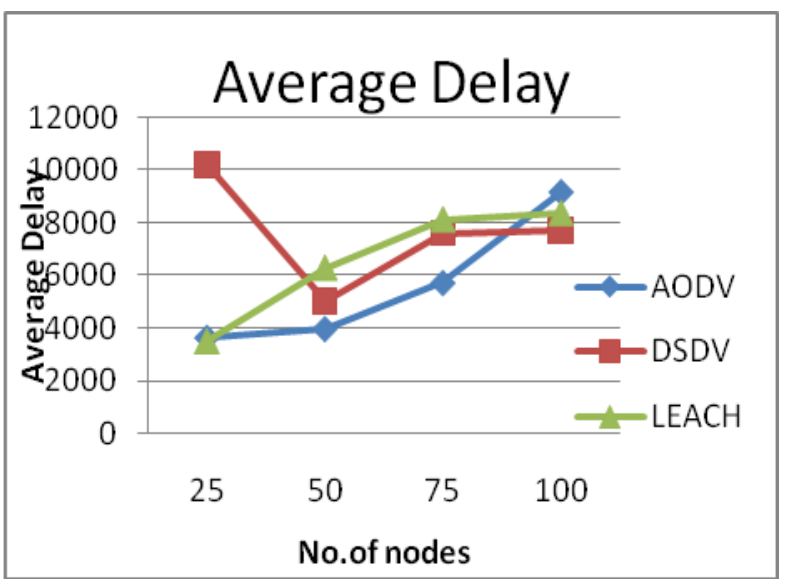

Figure 10: Three protocols of WSNs with respect to Average End-to-End Delay

\section{Results:}

1. From figure 10, we obtain that in AODV; the average endto-end delay becomes very high and increases when the number of nodes increases.

2. In DSDV, the average end-to-end delay becomes very high and increases when the number of node increases except at network size 25 .

3. In LEACH, the average end-to-end delay become very high and increases when the number of node increases.

4. The performance of LEACH is better with more number of nodes than in comparison with other two protocols. 


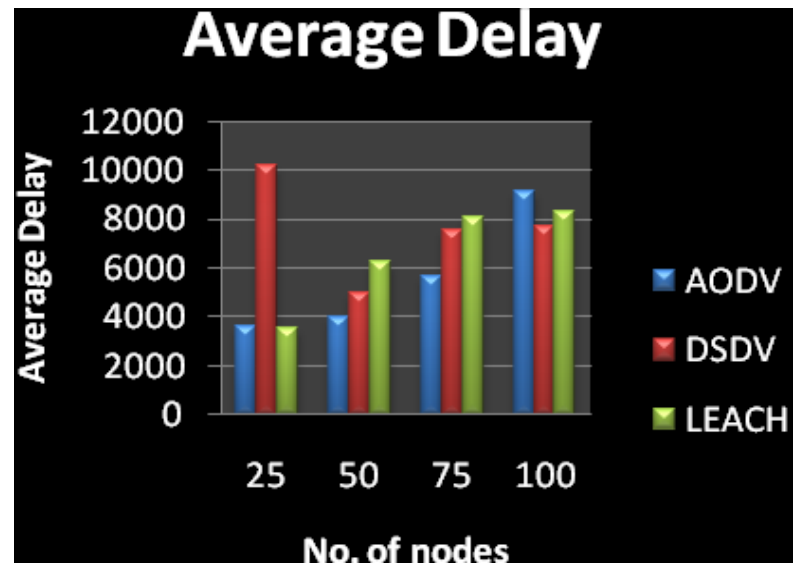

Figure 11: Comparison of the three protocols of WSNs with respect to Average End-to-End Delay

\section{CONCLUSION}

In this work, we focused on the routing problem in wireless sensor networks. We have presented an extensive simulation study to compare three on-demand protocols (AODV, DSDV and LEACH), using a variety of workloads such as packet delivery ratio, routing over head, throughput and average delay. According to practical results, the routing protocol AODV gives the better performance for both MANETs and WSNs. Our results indicate that AODV and LEACH both perform better but AODV is less reliable than LEACH because the result of AODV is fluctuated compare to LEACH.

\section{ACKNOWLEDGMENT}

To the almighty creation, we remain thankful for everything that we have. Our deep sense sincere regards profound indebtedness to all the faculty \& staff members of the Department of Electronics and Communication Engineering, National Institute of Technology Kurukshetra and Govind Ballabh Pant Engineering College, Pauri, for their cooperation in my work.

\section{REFERENCES}

[1] C.Y Chong, S.P.Kumar, "Sensor networks: evolution, opportunities, and challenges" ,IEEE Journals , Volume: 91 , Issue: 8,2003 , pp: 1247 - 1256

[2] G. Sharma,S. Bala, A.K Verma, "Comparison of Flooding and Directed Diffusion for Wireless Sensor Network", IEEE Confrence on India Conferences (INDICON),2009,pp 1 - 4

[3] I.F.Akyildiz, S. W. Sankarasubramaniam, E. Cayirci , “ $A$ survey on sensor networks" , IEEE Journal on Communication,Volume:40,Issue:8,2002,pp102-114

[4] K. Akkaya and M. Younis, "A Survey on Routing Protocols for Wireless Sensor Networks," Science Direct, Elsevier, Ad Hoc Networks, Volume: 3 Issue: 3, pp. 325-349, 2005.

[5] N. S. M. Usop, A. Abdullah, A. F. A. Abidin, "Performance evaluation of AODV, DSDV \& DSR routing protocol in grid environment" IJCSNS International Journal of Computer Science and Network Security, Volume:9,I ssue: 7, July 2009,pp 261-268.

[6] P.Nair ,H.Cam, S.Ozdemir and D. M Espda: "Energy efficient and secure pattern based data aggregation for wireless sensor networks" "IEEE Confrence on cations IEEE Sensors, Volume: 2,2003,pp732-736

[7] S.C.Jung, H.K.Choi, "Security in wireless sensor networks",IEEE Confrence onAdvanced Communication Technology, Volume:012009. pp 358 - 361

[8] W. Heinzelman, A. Chandrakasan and H. Balakrishnan, "Energy-Efficient Communication Protocol for Wireless Micro-sensor Networks," Proceedings of the 33rd Hawaii International Conference on System Sciences (HICSS '00), January. 2000. 